\title{
Autoimmune, neurological, and venous thromboembolic adverse events after immunisation of adolescent girls with quadrivalent human papillomavirus vaccine in Denmark and Sweden: cohort study \\ (c) (1) ()
}

\author{
Lisen Arnheim-Dahlström associate professor ${ }^{1}$, Björn Pasternak postdoctoral fellow ${ }^{2}$, Henrik \\ Svanström statistician $^{2}$, Pär Sparén professor ${ }^{1}$, Anders Hviid senior investigator ${ }^{2}$
}

${ }^{1}$ Department of Medical Epidemiology and Biostatistics, Karolinska Institutet, Stockholm 171 77, Sweden; ${ }^{2}$ Department of Epidemiology Research, Statens Serum Institut, Copenhagen, Denmark

\begin{abstract}
Objective To assess the risk of serious adverse events after vaccination of adolescent girls with quadrivalent human papillomavirus (qHPV) vaccine.

Design Register based cohort study.

Setting Denmark and Sweden, October 2006 to December 2010.

Participants 997585 girls aged 10-17, among whom 296826 received a total of 696420 qHPV vaccine doses.

Main outcome measures Incident hospital diagnosed autoimmune, neurological, and venous thromboembolic events (53 different outcomes) up to 180 days after each qHPV vaccine dose. Only events with at least five vaccine exposed cases were considered for further assessment. Rate ratios adjusted for age, country, calendar year, and parental country of birth, education, and socioeconomic status were estimated, comparing vaccinated and unvaccinated person time. For outcomes where the rate ratio was significantly increased, we regarded three criteria as signal strengthening: analysis based on 20 or more vaccine exposed cases (reliability), rate ratio 3.0 or more (strength), and significantly increased rate ratio in country specific analyses (consistency). We additionally assessed clustering of events in time and estimated rate ratios for a risk period that started on day 181 .

Results Among the 53 outcomes, at least five vaccine exposed cases occurred in 29 and these were analysed further. Whereas the rate ratios for 20 of 23 autoimmune events were not significantly increased, exposure to qHPV vaccine was significantly associated with Behcet's syndrome, Raynaud's disease, and type 1 diabetes. Each of these three outcomes fulfilled only one of three predefined signal strengthening
\end{abstract}

criteria. Furthermore, the pattern of distribution in time after vaccination was random for all three and the rate ratios for these outcomes in the period from day 181 after vaccination were similar to the rate ratios in the primary risk period. The rate ratios for five neurological events were not significantly increased and there were inverse associations with epilepsy (rate ratio $0.66,95 \%$ confidence interval 0.54 to 0.80 ) and paralysis $(0.56,0.35$ to 0.90$)$. There was no association between exposure to $\mathrm{GHPV}$ vaccine and venous thromboembolism $(0.86,0.55$ to 1.36$)$.

Conclusions This large cohort study found no evidence supporting associations between exposure to GHPV vaccine and autoimmune, neurological, and venous thromboembolic adverse events. Although associations for three autoimmune events were initially observed, on further assessment these were weak and not temporally related to vaccine exposure. Furthermore, the findings need to be interpreted considering the multiple outcomes assessed.

\section{Introduction}

Since the regulatory approval of first the quadrivalent human papillomavirus (qHPV) vaccine in 2006 and later the bivalent HPV vaccine, as of 2011 about 120 million doses have been distributed worldwide. ${ }^{1}$ The introduction of a new vaccine invariably puts focus not only on its effectiveness but also on its safety. From experience we know that adverse events, with onset shortly after the receipt of a vaccine, especially if these events are serious (for example, chronic immune mediated and neurological diseases), tend to be attributed to this exposure by pure temporal association. ${ }^{23}$ The likelihood of adverse events 
occurring in temporal association with exposure to vaccine will increase proportionally with vaccine coverage as a new vaccine is introduced. Concern about vaccine related adverse events has been identified as an important barrier to vaccination and one of the reasons for low HPV vaccine uptake in some settings. ${ }^{45}$ Concerns about autoimmune and neurological conditions being triggered by HPV vaccination may be fueled further by findings related to other vaccines, such as the reported association between adjuvanted influenza $\mathrm{A}(\mathrm{H} 1 \mathrm{~N} 1)$ pdm09 vaccine and narcolepsy in Sweden and Finland ${ }^{67}$ as well as a small but significantly increased risk of Guillain-Barré syndrome after influenza $\mathrm{A}(\mathrm{H} 1 \mathrm{~N} 1)$ pdm09 vaccination, according to a recent meta-analysis. ${ }^{8}$ Several potential mechanisms by which vaccines could induce or stimulate autoimmunity have been suggested, including molecular mimicry and bystander activation. ${ }^{2}$

Consequently, to acquire the best possible evidence of safety, adverse events of newly introduced vaccines such as the HPV vaccines need to be monitored continuously.

Most adverse events occurring after HPV vaccination of adolescent girls have been mild and temporary, such as fever, headache, and injection site reactions. ${ }^{9}{ }^{10}$ Pooled analyses of clinical trials have involved almost 12000 participants exposed to the qHPV vaccine ${ }^{11}$ and more than 16000 participants exposed to the bivalent HPV vaccine. ${ }^{12}$ Although these studies did not identify an increased risk of chronic or autoimmune diseases overall, they were not large enough to study individual conditions. An analysis of 12424 reports to the Vaccine Adverse Event Reporting System, among which 772 described serious events, identified disproportionate reporting of syncope and venous thromboembolic events but not other events, such as autoimmune conditions. ${ }^{13}$ However, analysis of data reported to passive surveillance can only identify potential risk signals and can neither estimate the risk relative to an unexposed population nor exclude risks with certainty. Sequential analyses of Vaccine Safety Datalink observational data within seven managed care organisations in the United States (600 558 qHPV vaccine doses) found no increased risk of eight prespecified outcomes, although a non-significantly increased relative risk of venous thromboembolism was observed..$^{14} \mathrm{~A}$ cohort study of 189629 women in two managed care organisations in California explored the risk of 16 autoimmune events and found a significantly increased rate ratio for Hashimoto's thyroiditis, although further assessment revealed no temporal relation or biological plausibility to support a true association. ${ }^{15}$ In this same cohort, no new safety concerns emerged when the risk of visits to an emergency department or admissions to hospital were evaluated for a wide range of health outcomes. ${ }^{16}$

Sweden and Denmark keep population based healthcare registers and thereby have unique opportunities to address the safety of HPV vaccination. In Denmark, we have previously described the incidence rates of anticipated immune mediated adverse events among adolescent girls in the period before the introduction of HPV vaccination. ${ }^{17}$ In the present study we identified potential safety signals after the introduction of qHPV vaccination in Denmark and Sweden by comparing incidence rates of several autoimmune, neurological, and venous thromboembolic adverse events between adolescent girls exposed and not exposed to the qHPV vaccine.

\section{Methods}

\section{Study population}

This register based cohort study of serious adverse events associated with the qHPV vaccine was based on individual level data from all 10 to 17 year old adolescent girls in Denmark and
Sweden, between 1 October 2006 and 31 December 2010. Every resident in both Denmark and Sweden has a unique personal identification number enabling individual level linkage between multiple registers. ${ }^{18}$ To define the study cohort in Sweden, we obtained information on birth date and date of death from the total population register and death register, respectively, from Statistics Sweden. To define the study cohort in Denmark, we used the Civil Registration System, which contains daily updated information on vital and demographic variables, such as birth date and place, and loss to follow-up due to emigration or disappearance from the registers, and death. ${ }^{19}$

\section{Vaccination}

The qHPV vaccine (Gardasil; Sanofi Pasteur MSD SNC, Lyon, France (in the United States: Merck, Whitehouse Station, NJ)) was marketed in Europe on 20 September 2006. In Sweden, the qHPV vaccine has been subsidised for 13-17 year old adolescent girls since May 2007 (inclusion of HPV vaccination in the national vaccination programme for 10-12 year old girls was initially planned for January 2010 but was postponed to January 2012 and then coupled with catch-up vaccination of 13-17 year old adolescent girls). In Denmark, the qHPV vaccine has been included in the national vaccination programme since January 2009 for 12 year old girls, with catch-up vaccination of 13-15 year olds from October 2008

We obtained information on exposure to HPV vaccine in Denmark from the childhood vaccination database at Statens Serum Institut. ${ }^{20}$ This database is continually updated from National Health Insurance data obtained from the National Board of Health. General practitioners in Denmark carry out all immunisations in the childhood vaccination programme and are reimbursed for reporting each instance to the National Health Insurance. Reimbursement takes place only after the general practitioner has submitted a form that details the vaccine, the date it was administered, and the personal identification number of the recipient. Therefore the database is thought to be close to complete for vaccines administered through the national programme. Because HPV vaccination was also available outside the national programme in the study period, we retrieved additional vaccination data from the national prescription register, which contains individual level information on all prescriptions filled at all Danish pharmacies. This includes the personal identification number of the recipient, the date the prescription was dispensed, and the Anatomic Therapeutic Chemical (ATC) code. ${ }^{21}$ The ATC code used to identify the qHPV vaccine was J07BM01. In Sweden, correspondingly, we obtained information on vaccination status from Svevac and the drug prescription register. Svevac is a national HPV vaccination register, established in 2006 and held by the Swedish Institute for Communicable Disease. Healthcare staff who administer the vaccines report to Svevac on a voluntary basis, and the register has an estimated completeness of about $80 \% .^{22}$ The drug prescription register contains all prescription drugs dispensed at pharmacies in Sweden since 1 July 2005. ${ }^{23}$ Adolescent girls aged between 13 and 17 years received subsidised HPV vaccination and vaccination had to be prescribed and expedited at a pharmacy, thereby generating a register entry in the drug prescription register. The register is held by the National Board of Health and Welfare. For adolescent girls aged 13-17 years it is assumed that almost $100 \%$ of administered HPV vaccine doses are registered in the drug prescription register. 


\section{Outcomes}

We identified data on predefined adverse events from the national patient registers in both countries using ICD-10 codes (international classification of diseases, 10th revision). The patient registers include nationwide individual level information on dates of hospital contact and doctor assigned diagnoses according to the international classification of diseases. ${ }^{24} \mathrm{We}$ did not have information on outcomes from primary healthcare. The Danish patient register was established in 1977, has included both inpatients and outpatients since 1994, and has been using ICD-10 codes since 1995, whereas the Swedish patient register has had nationwide coverage since 1987 , has included both inpatients and outpatients since 2001, and has used ICD-10 codes since $1997 .{ }^{25}$ We predefined several serious adverse outcome events, as identified from records of inpatient admissions and hospital outpatient and emergency department visits, based on our earlier study of autoimmune events, ${ }^{17}$ and we added several neurological events. We also included venous thromboembolism because it represents a potential adverse event. ${ }^{13}{ }^{14}$ The included outcomes are all well defined diseases. In total, we assessed 53 outcomes (see supplementary table 1 for all included outcome events, with ICD-10 codes).

\section{Covariate information}

From Statistics Denmark and Statistics Sweden we obtained data on parental educational level, country of birth, and socioeconomic status. We identified the parents from the Danish Civil Registration System and Swedish multigeneration register.

\section{Statistical analyses}

Adolescent girls were followed from age 10 years or 1 October 2006, whichever came latest, and until either the occurrence of an adverse event, receipt of bivalent HPV vaccine (Cervarix, GlaxoSmithKline Biologicals; Rixensart, Belgium; ATC code J07BM02), death, disappearance from the registers, emigration (this information was only available for Denmark), 18th birthday, or end of follow-up (31 December 2010). We aggregated the resulting person years of follow-up with counts of outcome events according to qHPV vaccine exposure status and analysed these using Poisson regression (log-linear regression of the counts using the logarithm of follow-up time as offset). This produced incidence rate ratios according to qHPV exposure status. Exposure to the qHPV vaccine was a time varying variable; thus adolescent girls could contribute person time to the study first as unvaccinated and later as vaccinated, but once vaccinated the girls could not be put into the unvaccinated category again. All individual outcomes were treated as separate analyses, and for each specific analysis girls were eligible only if free from the outcome event before entry to the cohort. Estimates were adjusted for country, age in two year categories, calendar year, parental educational level (highest attained level of either parent classified as: primary school (nine years) or shorter; secondary school (12 years); short tertiary education; and medium or long tertiary education), parental country of birth (categories: both parents, one parent, or no parent born in Scandinavia), and paternal socioeconomic status (categories: employment with basic, unknown, or no qualification; employment with medium level or high level qualifications; self employed; and not in labour market).

For all autoimmune and neurological outcomes, we defined the period at risk as 180 days after exposure to vaccine. This period was chosen to allow for the insidious onset of the diseases studied and because diagnostic investigations may take time; in a recent study of autoimmune outcomes after qHPV vaccination, the median time between first symptoms and diagnosis was 23 days (interquartile range 2-59 days). ${ }^{15}$ For venous thromboembolism, the period at risk was 90 days after vaccination. This was regarded as the maximal period where an acute event could be plausibly related to vaccination; furthermore, the mean time between vaccination and diagnosis of thromboembolism among cases reported to the Vaccine Adverse Event Reporting System was 42 days. ${ }^{13}$

Because we acquired data on vaccine exposure from two sources that were partly overlapping - that is, some of the girls had both filled prescriptions and were registered in one of the vaccination databases-we applied an algorithm to harmonise the data and define dates of vaccination. Essentially this algorithm removed double data entries and doses appearing beyond the three dose schedule. Furthermore, on the basis of data from girls with both filled prescriptions and register entries in vaccination databases, it was established that the median lag between the dispensing of a prescription and the date of vaccination as registered in the vaccination databases was two days. Consequently, for vaccine doses that were defined by prescriptions alone, the date of exposure was defined as the date of filling the prescription plus two days.

As the recommended qHPV vaccine schedule includes three doses given at 0,2 , and 6 months, any girl could contribute up to three doses in the analyses; we counted exposed person time from the date the vaccine was administered, and each dose contributed up to 180 days (autoimmune and neurological events) of follow-up or up to 90 days (venous thromboembolism) of follow-up (fig $1 \Downarrow$ ). We used SAS statistical software version 9.3 (SAS Institute, Cary, NC).

\section{Analytical strategy}

Given that a large number of serious adverse events were assessed and consequently there was a high probability of chance findings, we used the following predefined criteria for the analysis of data. As the first criterion, and in the interest of obtaining relatively reliable rate ratios, for any further assessment to take place we considered only outcomes with at least five vaccine exposed cases during the predefined period at risk. To be regarded as a safety signal, the rate ratio for an outcome with at least five vaccine exposed cases had to be significantly increased (lower bound of $95 \%$ confidence interval $>1.0$ ). We regarded three criteria as signal strengthening: analysis based on 20 or more vaccine exposed cases (reliability of analysis); a rate ratio of 3.0 or more (strength of association); and significantly increased rate ratios in both countries when analysed separately (consistency). For outcomes where the rate ratio was significantly increased, we additionally assessed clustering of events in time by plotting cases according to time since exposure to the vaccine and estimated rate ratios for a risk period that started on day 181 .

\section{Results Cohort}

The study cohort included 997585 girls of whom 296826 (29.8\%) received at least one dose of the qHPV vaccine (table $1 \Downarrow$ ). Among the vaccinated girls, 238608 ( $80.4 \%$ of vaccinated girls and $23.9 \%$ of total study cohort) received the second dose and 160986 (54.2\% of vaccinated girls and $16.1 \%$ of total study cohort) received the third. Overall, $696420 \mathrm{qHPV}$ vaccine doses were administered. During follow-up, 1322 girls received the bivalent HPV vaccine and hence were censored. 


\section{Outcome events}

Of the 53 assessed outcomes, 29 fulfilled the criterion for further analysis ( $\geq 5$ vaccine exposed cases within the predefined risk periods after vaccination) whereas 24 did not (see supplementary table 2). Table $2 \Downarrow$ and figure $2 \Downarrow$ show crude incidence rates and adjusted rate ratios, respectively, according to exposure status of the qHPV vaccine for the 29 analysed outcomes.

\section{Autoimmune events}

The rate ratios for 20 of the 23 analysed autoimmune outcomes were not significantly increased. Exposure to qHPV vaccine was significantly associated with Behcet's syndrome (rate ratio $3.37,95 \%$ confidence interval 1.05 to10.80), Raynaud's disease (1.67, 1.14 to 2.44$)$, and type 1 diabetes $(1.29,1.03$ to 1.62$)$.

\section{Neurological events}

The rate ratios were not significantly increased for any of the five analysed neurological outcomes. For two of these outcomes, epilepsy and paralysis, the rate ratios were significantly decreased.

\section{Venous thromboembolism}

The rate ratio for the association between exposure to qHPV vaccine and venous thromboembolism was 0.86 (0.55 to 1.36$)$.

\section{Evaluation of safety signals}

Each of the three autoimmune outcome events where the rate ratios were significantly increased was assessed using the predefined signal strengthening criteria. For each of these three outcomes, one of the three signal strengthening criteria was fulfilled (table $3 \Downarrow$ ). For Raynaud's disease and type 1 diabetes, the fulfilled criterion was that the analysis was based on 20 or more vaccine exposed cases. For Behcet's syndrome, the criterion was that the rate ratio was 3.0 or above.

Subsequently, for each of the three outcome events with a significantly increased rate ratio within 180 days after qHPV vaccination, we estimated rate ratios for a later period. Starting from day 181 after vaccination and onwards, the rate ratios were 3.18 (95\% confidence interval 0.83 to 12.19 ) for Behcet's syndrome, 1.50 (0.95 to 2.37) for Raynaud's disease, and 1.18 ( 0.91 to 1.55 ) for type 1 diabetes (see supplementary table 3 ).

Visual inspection of the temporal distribution of cases revealed no distinct pattern in time for Raynaud's disease and type 1 diabetes (fig $3 \Downarrow$ ). For Behcet's syndrome, this analysis was inconclusive owing to the low number of cases.

\section{Sensitivity analysis}

Because of the possible delay between disease onset and diagnosis, the selected risk period of 180 days might not have captured narcolepsy events adequately. Therefore we did a sensitivity analysis with the risk period starting from day 181; the rate ratio was 0.64 (95\% confidence interval 0.26 to 1.57 ; see supplementary table 4$)$.

\section{Discussion}

This population based study included all adolescent girls aged between 10 and 17 years in Denmark and Sweden who received quadrivalent human papillomavirus (qHPV) vaccine in the first four years after its licensure. Overall, the findings of this study, which were based on nearly one million girls and 700000 vaccine doses, were reassuring for autoimmune, neurological, and venous thromboembolic events after qHPV vaccination.
Among 40 predefined autoimmune outcomes, five or more vaccine exposed cases occurred in 23, and these were analysed further. Exposure to qHPV vaccine was not significantly associated with 20 of these autoimmune outcome events. However, the rate ratios were significantly increased for Behcet's syndrome, Raynaud's disease, and type 1 diabetes. According to the predefined analytical strategy, we assessed the strength of the observed safety signal for these outcome events; for each of the three outcomes, only one of three predefined signal strengthening criteria was met. Furthermore, the rate ratios for the association between exposure to qHPV vaccine and each of these three outcome events in the period starting on day 181 after vaccination and beyond were similar to the rate ratios in the primary period at risk (180 days). Additionally, on visual inspection, the distribution of cases exhibited a random pattern. The initially observed associations thus need to be interpreted with several factors considered: the overall number of outcomes included in the study and hence the possibility of chance findings; the relative weakness of these signals, as per our evaluation of predefined signal strengthening criteria; and the biological implausibility of events occurring without a clear temporal pattern in relation to the exposure. On this basis, although significantly increased rate ratios were initially observed for three outcomes, it can be concluded that after further assessment no consistent evidence for a causal association was found.

\section{Relation to other studies}

These findings corroborate those from a cohort study of 189 629 women in two managed care organisations in California, which found no safety signal when investigating the risk of 16 autoimmune events. ${ }^{15}$ That study did find an inverse association between exposure to qHPV vaccine and type 1 diabetes (rate ratio $0.57,95 \%$ confidence interval 0.47 to 0.73 ), lending further support to the conclusion that the initial signal for type 1 diabetes observed in our study might be a false positive.

Of the 12 neurological outcomes assessed in our study, five fulfilled the criterion for further analyses. The rate ratio was not significantly increased for any of these five outcomes, and both epilepsy and paralysis were inversely associated with exposure to qHPV vaccine. Not at least given that the Vaccine Safety Datalink study of qHPV vaccine related adverse events reported a relative risk of 1.02 for seizures,${ }^{14}$ the observed inverse association for this outcome in our study is most likely due to chance. This underlines the need to interpret the findings from our study taking into account the large number of comparisons made and the need for ongoing monitoring in independent populations.

Analysis of data reported to the Vaccine Adverse Event Reporting System revealed disproportionate reporting of venous thromboembolism. ${ }^{13}$ A study by the Vaccine Safety Datalink, which involved eight outcomes, identified a non-significantly increased relative risk (1.98) of venous thromboembolism; medical record review could confirm five of the eight cases identified from databases using international classification of diseases codes, and all five had known risk factors for venous thromboembolism. ${ }^{14}$ In our analysis, based on 21 vaccine exposed cases, there was no significant association with venous thromboembolism within 90 days after exposure to qHPV vaccine. These results are corroborated by a study in two managed care organisations in California that did not find evidence of an association between qHPV vaccination and venous thromboembolism when assessing all events in emergency department and admissions to hospital using international classification of diseases codes. ${ }^{16}$ 


\section{Strengths and weaknesses of this study}

Our study has strengths and limitations. We analysed nationwide data from Denmark and Sweden, which permitted a large sample size; this was advantageous because many of the outcomes studied are rare and therefore not possible to study in a clinical trial. Furthermore, the use of individually linked data allowed the identification of truly incident cases. Additionally, the inclusion of entire populations in the cohort allowed the rates among those exposed to vaccine to be compared directly with the true national background rates. ${ }^{27}$ Although Denmark and Sweden are alike in many respects, including having similar healthcare systems and universal access to healthcare, differences in diagnostic coding practices might have influenced the ascertainment of cases. This is, however, unlikely for relatively well defined diseases. Our case definition was based on hospital diagnoses, which likely captured the majority of cases because most adolescent girls with the severe conditions studied are under specialised paediatric care (this type of care is only available from hospitals in Denmark and Sweden), at the very least during the diagnostic phase.

Dates of onset of symptoms or disease were not available for this study, which instead relied on dates of diagnoses to define the index dates of outcome events. It is therefore possible that a proportion of events attributed to vaccine exposure in the analyses had symptom onset before the vaccine was administered, and similarly, that a proportion of events had symptom onset within the risk period but were diagnosed later on and were thus not attributed to vaccine exposure in the analyses. To accommodate the time delay between first symptom and diagnosis we used a 180 day risk period for autoimmune and neurological outcomes, which is longer than in some studies (for example, studies of Guillain-Barré syndrome after vaccination typically have a risk window of 42 days), but consistent with other studies of autoimmune disease after HPV vaccination. ${ }^{15}$ However, it may have been too short to capture diseases with a more insidious onset. Because the latter may be the case for narcolepsy, we conducted a sensitivity analysis with a risk window starting on day 181 after vaccination; no significant association was observed with narcolepsy.

The vaccine coverage was $49 \%$ in Denmark and $18 \%$ in Sweden. This difference between the countries may reflect the fact that during the study period the qHPV vaccine had been introduced in the national childhood vaccination programme in Denmark but not in Sweden. The statistical models included adjustment for age, country, calendar year, and parental educational level, country of birth, and socioeconomic status. However, we could not obtain information on other potential risk factors such as smoking as this information is not recorded in any nationwide register. This may represent a source of residual confounding. In particular, given an overall $30 \%$ vaccine coverage, adolescent girls who did receive the vaccine may be selected individuals and could be at differential risk of certain outcomes or be more or less likely to consult healthcare for health problems. Self controlled case series analysis ${ }^{28}$ could have tackled some of the potential problems from confounding. However, as the aim of this study was signal detection and strengthening and the method chosen is computationally efficient for analysing a large number of outcomes, the self controlled case series method is data intensive and would be cumbersome when studying a large number of outcomes. Furthermore, the self controlled case series becomes problematic when onset of disease is not known to any great extent (which is the case, for example, for narcolepsy, which normally has a long incubation period).

An unmasking phenomenon has been described in vaccine safety research. ${ }^{26}$ This refers to the fact that the vaccination visit provides an opportunity to evaluate symptoms that may not have been evaluated otherwise and, hence, that those vaccinated may be more likely to have certain disorders diagnosed; this would bias results towards increased risk attributed to vaccination. This phenomenon is unlikely for diseases with relatively prominent and well recognised symptoms, such as type 1 diabetes, but is plausible for diseases that may present with obscure symptoms or that initially may be interpreted by the patient as normal variation, such as Raynaud's disease. Although the results from this study are possibly generalisable to comparable populations that are of similar age, they cannot be directly extrapolated to adults. Furthermore, the results should not be inferred to the bivalent HPV vaccine, because the constituents of the vaccine differ.

\section{Conclusion}

This cohort study of about one million adolescent girls aged 10 to 17 years utilised routine healthcare data from two Scandinavian countries to identify potential serious adverse events during the first four years after the qHPV vaccine was marketed. While the study expands on the current safety information of the qHPV vaccine by systematically assessing a range of serious adverse outcomes, the results need to be interpreted cautiously considering the large number of statistical tests performed; as well as the chance of false positive findings, true associations may have been missed. Although significantly increased rate ratios were initially observed for three outcomes, further assessment showed no consistent evidence for a plausible association; firstly, these risk signals were relatively weak, as assessed by prespecified criteria, and, secondly, no temporal relation between vaccine exposure and outcome was evident. Thus, this study identified no safety signals with respect to autoimmune, neurological, and venous thromboembolic events after the qHPV vaccine had been administered. Nevertheless, these findings need to be confirmed in studies with longer follow-up time, validation of outcomes, and data on time of onset of disease. Further monitoring of HPV vaccine safety is warranted in other populations when use and coverage has increased.

We thank Jonas Hällgren for data administration, Karin Sundström for valuable discussion on the findings in this study, and the Swedish Institute for Communicable Diseases for contributing with HPV vaccination data from the Svevac register.

Contributors: BP, HS, and AH created the database and performed the statistical analyses. LAD and BP drafted the manuscript. All authors actively participated in study design, interpretation and discussion of the results, revision of the manuscript, and approval of the final version of the manuscript. $\mathrm{AH}$ is the guarantor.

Funding: This study was supported by a grant from the Swedish Foundation for Strategic Research and the Danish Medical Research Council. The funding bodies had no role in the study design; the collection, analysis, and interpretation of the data; the writing of the article; and the decision to submit it for publication. All authors are independent from the funding agencies.

Competing interests: All authors have completed the ICMJE uniform disclosure form at www.icmje.org/coi_disclosure.pdf (available on request from the corresponding author) and declare: no support from any organisation for the submitted work; LAD and PS are and have been involved in other studies with unconditional grants from GlaxoSmithKline, Sanofi Pasteur MSD, and Merck; and no other relationships or activities that could appear to have influenced the submitted work. 


\section{What is already known on this topic}

Vaccines against human papillomavirus (HPV) have been available since 2006

Clinical trials and post-licensure studies from the United States have not identified any increase in the risk of serious adverse events after vaccination

\section{What this study adds}

This European cohort study found no evidence supporting associations between exposure to qHPV vaccine and autoimmune, neurological, and venous thromboembolic adverse events in almost one million adolescent girls

Ethical approval: This study was approved by the regional ethical review committee in Stockholm, Sweden, and by the Danish Data Protection Agency. Ethical approval is not required for register based research in Denmark.

Declaration of transparency: The lead author affirms that this manuscript is an honest, accurate, and transparent account of the study being reported; that no important aspects of the study have been omitted; and that any discrepancies from the study as planned (and, if relevant, registered) have been explained.

Data sharing: No additional data available.

1 Medical Products Agency. Vaccination against human papillomavirus (HPV) vaccine Gardasil and Cervarix. 2012. [In Swedish.] www.lakemedelsverket.se/OVRIGA-SIDOR/ HPV-vaccinering/.

2 Wraith DC, Goldman M, Lambert PH. Vaccination and autoimmune disease: what is the evidence? Lancet 2003;362:1659-66.

3 Siegrist CA. Autoimmune diseases after adolescent or adult immunization: what should we expect? CMAJ 2007;177:1352-4.

4 Trim K, Nagji N, Elit L, Roy K. Parental knowledge, attitudes, and behaviours towards human papillomavirus vaccination for their children: a systematic review from 2001 to 2011. Obstet Gynecol Int 2012;2012:921236

5 Laz TH, Rahman M, Berenson AB. An update on human papillomavirus vaccine uptake among 11-17 year old girls in the United States: national health interview survey, 2010. Vaccine 2012;30:3534-40.

6 Nohynek H, Jokinen J, Partinen M, Vaarala O, Kirjavainen T, Sundman J, et al. AS03 adjuvanted $\mathrm{AH} 1 \mathrm{~N} 1$ vaccine associated with an abrupt increase in the incidence of childhood narcolepsy in Finland. PLoS One 2012;7:e33536.

7 Medical Products Agency. Occurrence of narcolepsy with cataplexy among children and adolescents in relation to the H1N1 pandemic and Pandemrix vaccinations. Secondary occurrence of narcolepsy with cataplexy among children and adolescents in relation to the H1N1 pandemic and Pandemrix vaccinations 2011. www.lakemedelsverket.se/upload/ nyheter/2011/Fallinventeringsrapport_pandermrix_110630.pdf.

8 Salmon DA, Proschan M, Forshee R, Gargiullo P, Bleser W, Burwen DR, et al. Association between Guillain-Barre syndrome and influenza A (H1N1) 2009 monovalent inactivated vaccines in the USA: a meta-analysis. Lancet 2013;381:1461-8.

9 Lu B, Kumar A, Castellsague X, Giuliano AR. Efficacy and safety of prophylactic vaccines against cervical HPV infection and diseases among women: a systematic review and meta-analysis. BMC Infect Dis 2011;11:13.

10 Van Klooster TM, Kemmeren JM, van der Maas NA, de Melker HE. Reported adverse events in girls aged 13-16 years after vaccination with the human papillomavirus (HPV)-16/18 vaccine in the Netherlands. Vaccine 2011;29:4601-7.

11 Block SL, Brown DR, Chatterjee A, Gold MA, Sings HL, Meibohm A, et al. Clinical trial and post-licensure safety profile of a prophylactic human papillomavirus (types $6,11,16$ and 18) I1 virus-like particle vaccine. Pediatr Infect Disease J 2010;29:95-101.

12 Descamps D, Hardt K, Spiessens B, Izurieta P, Verstraeten T, Breuer T, et al. Safety of human papillomavirus (HPV)-16/18 AS04-adjuvanted vaccine for cervical cancer prevention: a pooled analysis of 11 clinical trials. Hum Vaccin 2009;5:332-40.
13 Slade BA, Leidel L, Vellozzi C, Woo EJ, Hua W, Sutherland A, et al. Postlicensure safety surveillance for quadrivalent human papillomavirus recombinant vaccine. JAMA 2009;302:750-7.

14 Gee J, Naleway A, Shui I, Baggs J, Yin R, Li R, et al. Monitoring the safety of quadrivalent human papillomavirus vaccine: findings from the Vaccine Safety Datalink. Vaccine 2011;29:8279-84

15 Chao C, Klein NP, Velicer CM, Sy LS, Slezak JM, Takhar H, et al. Surveillance of autoimmune conditions following routine use of quadrivalent human papillomavirus vaccine. J Intern Med 2012;271:193-203.

16 Klein NP, Hansen J, Chao C, Velicer C, Emery M, Slezak J, et al. Safety of quadrivalen human papillomavirus vaccine administered routinely to females. Arch Pediatr Adolesc Med 2012:166:1140-8.

17 Callreus T, Svanstrom H, Nielsen NM, Poulsen S, Valentiner-Branth P, Hviid A. Human papillomavirus immunisation of adolescent girls and anticipated reporting of immune-mediated adverse events. Vaccine 2009;27:2954-8.

18 Ludvigsson JF, Otterblad-Olausson P, Pettersson BU, Ekbom A. The Swedish personal identity number: possibilities and pitfalls in healthcare and medical research. Eur $J$ Epidemiol 2009;24:659-67.

19 Pedersen CB, Gotzsche H, Moller JO, Mortensen PB. The Danish Civil Registration System. A cohort of eight million persons. Dan Med Bull 2006;53:441-9.

20 Hviid A. Postlicensure epidemiology of childhood vaccination: the Danish experience. Expert Rev Vaccines 2006;5:641-9.

21 Kildemoes HW, Sorensen HT, Hallas J. The Danish National Prescription Registry. Scand $\checkmark$ Public Health 2011;39:38-41.

22 Leval A, Herweijer E, Ploner A, Eloranta S, Fridman Simard S, Dillner J,et al. Quadrivalent HPV-vaccine effectiveness on genital warts: a population-based study in over 2.2 million women including dose evaluation. J Natl Cancer Inst 2013 (in press).

23 Wettermark B, Hammar N, Fored CM, Leimanis A, Otterblad Olausson P, Bergman U, et al. The new Swedish Prescribed Drug Register-opportunities for pharmacoepidemiological research and experience from the first six months. Pharmacoepidemiol Drug Safety 2007;16:726-35

24 Lynge E, Sandegaard JL, Rebolj M. The Danish National Patient Register. Scand J Public Health 2011;39:30-3.

25 Ludvigsson JF, Andersson E, Ekbom A, Feychting M, Kim JL, Reuterwall C, et al. External review and validation of the Swedish national inpatient register. BMC Public Health 2011;11:450.

26 Chao C, Jacobsen SJ. Evaluation of autoimmune safety signal in observational vaccine safety studies. Hum Vaccin Immunother 2012;8:1302-4.

27 Rasmussen TA, Jorgensen MR, Bjerrum S, Jensen-Fangel S, Stovring H, Ostergaard L, et al. Use of population based background rates of disease to assess vaccine safety in childhood and mass immunisation in Denmark: nationwide population based cohort study. BMJ 2012:345:e5823.

28 Farrington $\mathrm{CP}$, Nash J, Miller E. Case series analysis of adverse reactions to vaccines: a comparative evaluation. Am J Epidemiol 1996;143:1165-73.

Accepted: 28 August 2013

\section{Cite this as: BMJ 2013;347:f5906}

This is an Open Access article distributed in accordance with the Creative Commons Attribution Non Commercial (CC BY-NC 3.0) license, which permits others to distribute, remix, adapt, build upon this work non-commercially, and license their derivative works on different terms, provided the original work is properly cited and the use is non-commercial. See: http://creativecommons.org/licenses/by-nc/3.0/. 


\section{Tables}

Table 1| Descriptive characteristics of adolescent girls aged 10-17 years included in cohort, Denmark and Sweden, October 2006-December 2010. Values are numbers (percentages) unless stated otherwise

\begin{tabular}{lccc} 
Characteristics & Overall (n=997 585) & Denmark (n=387 294) & Sweden (n=610 291) \\
Person years of follow-up & 2797701 & 1090515 & 1707186 \\
\hline Mean (SD) age at study entry (years) & $12.8(2.7)$ & $12.5(2.6)$ & $12.9(2.7)$ \\
\hline Year of study entry: & & & \\
\hline 2006 & $700156(70.2)$ & $260849(67.4)$ & $439307(72.0)$ \\
\hline 2007 & $74809(7.5)$ & $32044(8.3)$ & $42765(7.0)$ \\
\hline 2008 & $73653(7.4)$ & $31307(8.1)$ & $42346(6.9)$ \\
\hline 2009 & $73909(7.4)$ & $31439(8.1)$ & $42470(7.0)$ \\
\hline 2010 & $75058(7.5)$ & $31655(8.2)$ & $43403(7.1)$ \\
\hline Exposed to quadrivalent human papillomavirus vaccine & & \\
\hline Mean (SD) age at vaccination (years) & $14.6(1.7)$ & $14.0(1.6)$ & $15.7(1.4)$ \\
\hline Total vaccine doses, No (\% of total No in cohort): & 696420 & 409724 & 286696 \\
\hline Dose 1 & $296826(29.8)$ & $188053(48.6)$ & $108773(17.8)$ \\
\hline Dose 2 & $238608(23.9)$ & $139861(36.1)$ & $98747(16.2)$ \\
\hline Dose 3 & $160986(16.1)$ & $81810(21.1)$ & $79176(13.0)$ \\
\hline Year of first vaccine dose, No (\% of vaccinated): & & & \\
\hline 2006 & $426(0.1)$ & $248(0.1)$ & $178(0.2)$ \\
\hline 2007 & $22943(7.7)$ & $6280(3.3)$ & $16663(15.3)$ \\
\hline 2008 & $41799(14.1)$ & $12314(6.5)$ & $29485(27.1)$ \\
\hline 2009 & $170830(57.6)$ & $133571(71.0)$ & $37259(34.3)$ \\
\hline 2010 & $60828(20.5)$ & $35640(19.0)$ & $25188(23.2)$ \\
\hline Data from vaccination registers & $572696(82.2)$ & $351804(85.9)$ & $220892(77.0)$ \\
\hline Data from prescription registers & $123724(17.8)$ & $57920(14.1)$ & $65804(23.0)$ \\
\hline & &
\end{tabular}

Because of rounding, percentages may not total 100 . 
Table 2| Rates of adverse events according to quadrivalent human papillomavirus (qHPV) vaccination status, cohort of adolescent girls aged 10-17 years in Denmark and Sweden, October 2006-December 2010

Unvaccinated

Adverse events

Person years No of events Incidence rate* $(95 \% \mathrm{Cl})$
Within 180 days after qHPV vaccine exposure

Person years No of events Incidence rate* $(95 \% \mathrm{Cl})$

Autoimmune

Thyroid:

\begin{tabular}{|c|c|c|c|c|c|c|}
\hline Graves' disease & 2373554 & 237 & 9.99 (8.79 to 11.34$)$ & 229914 & 27 & 11.74 (8.05 to 17.12$)$ \\
\hline Hashimoto's thyroiditis & 2371866 & 560 & 23.61 (21.73 to 25.65$)$ & 229751 & 50 & 21.76 (16.49 to 28.71$)$ \\
\hline Other hyperthyroidism & 2373629 & 250 & 10.53 (9.30 to 11.92$)$ & 229946 & 23 & 10.00 (6.65 to 15.05$)$ \\
\hline Hypothyroidism & 2368919 & 1018 & 42.97 (40.41 to 45.70$)$ & 229563 & 79 & 34.41 (27.60 to 42.90$)$ \\
\hline \multicolumn{7}{|l|}{ Gastrointestinal: } \\
\hline Coeliac disease & 2358918 & 1413 & 59.90 (56.86 to 63.11$)$ & 228820 & 107 & 46.76 (38.69 to 56.52 ) \\
\hline Crohn's disease & 2372337 & 539 & 22.72 (20.88 to 24.72$)$ & 229825 & 47 & 20.45 (15.37 to 27.22$)$ \\
\hline Ulcerative colitis & 2373288 & 350 & $14.75(13.28$ to 16.38$)$ & 229889 & 35 & $15.22(10.93$ to 21.20$)$ \\
\hline Pancreatitis & 2374129 & 103 & 4.34 (3.58 to 5.26 ) & 230004 & 10 & 4.35 (2.34 to 8.08 ) \\
\hline \multicolumn{7}{|l|}{ Muscoloskeletal or systemic: } \\
\hline Ankylosing spondylitis & 2374065 & 93 & 3.92 (3.20 to 4.80 ) & 230001 & 8 & 3.48 (1.74 to 6.96$)$ \\
\hline Behcet's syndrome & 2374464 & 13 & 0.55 (0.32 to 0.94$)$ & 230025 & 5 & 2.17 (0.90 to 5.22$)$ \\
\hline Henoch-Schönlein's purpura & 2369280 & 203 & 8.57 (7.47 to 9.83$)$ & 229365 & 17 & 7.41 (4.61 to 11.92$)$ \\
\hline Juvenile arthritis & 2366484 & 861 & 36.38 (34.03 to 38.90$)$ & 229202 & 86 & 37.52 (30.37 to 46.35$)$ \\
\hline Myositis & 2373974 & 84 & 3.54 (2.86 to 4.38$)$ & 229988 & 8 & 3.48 (1.74 to 6.96$)$ \\
\hline Rheumatoid arthritis & 2373763 & 216 & $9.10(7.96$ to 10.40$)$ & 229943 & 27 & $11.74(8.05$ to 17.12$)$ \\
\hline Systemic lupus erythematosus & 2374231 & 74 & 3.12 (2.48 to 3.91$)$ & 230005 & 11 & 4.78 (2.65 to 8.64$)$ \\
\hline Vasculitis, unspecified & 2373826 & 89 & 3.75 (3.05 to 4.61$)$ & 229959 & 14 & 6.09 (3.61 to 10.28$)$ \\
\hline \multicolumn{7}{|l|}{ Haematological: } \\
\hline $\begin{array}{l}\text { Idiopathic thrombocytopenic } \\
\text { purpura }\end{array}$ & 2373040 & 107 & 4.51 (3.73 to 5.45$)$ & 229896 & 14 & 6.09 (3.61 to 10.28$)$ \\
\hline \multicolumn{7}{|l|}{ Dermatological: } \\
\hline Erythema nodosum & 2373608 & 163 & 6.87 (5.89 to 8.01$)$ & 229935 & 19 & 8.26 (5.27 to 12.95$)$ \\
\hline Localised scleroderma & 2374016 & 88 & 3.71 (3.01 to 4.57 ) & 229976 & 6 & 2.61 (1.17 to 5.81$)$ \\
\hline Psoriasis & 2368423 & 1091 & 46.06 (43.41 to 48.88 ) & 229540 & 80 & 34.85 (27.99 to 43.39$)$ \\
\hline Vitiligo & 2372765 & 310 & 13.06 (11.69 to 14.60$)$ & 229886 & 24 & 10.44 (7.00 to 15.58$)$ \\
\hline \multicolumn{7}{|l|}{ Miscellaneous } \\
\hline Raynaud's disease & 2373798 & 218 & $9.18(8.04$ to 10.49$)$ & 229939 & 37 & 16.09 (11.66 to 22.21$)$ \\
\hline Type 1 diabetes & 2363153 & 975 & 41.26 (38.75 to 43.93$)$ & 228965 & 99 & 43.24 (35.51 to 52.65$)$ \\
\hline \multicolumn{7}{|l|}{ Neurological } \\
\hline Bell's palsy & 2370195 & 480 & 20.25 (18.52 to 22.15 ) & 229675 & 41 & 17.85 (13.14 to 24.24$)$ \\
\hline Epilepsy & 2351894 & 1701 & 72.32 (68.97 to 75.84 ) & 227897 & 116 & 50.90 (42.43 to 61.06$)$ \\
\hline Narcolepsy & 2374402 & 43 & 1.81 (1.34 to 2.44$)$ & 230018 & 6 & $2.61(1.17$ to 5.81$)$ \\
\hline Optical neuritis & 2374273 & 61 & 2.57 (2.00 to 3.30$)$ & 230013 & 6 & 2.61 (1.17 to 5.81$)$ \\
\hline Paralysis & 2367206 & 302 & $12.76(11.40$ to 14.28$)$ & 229574 & 20 & $8.71(5.62$ to 13.50$)$ \\
\hline Venous thromboembolism $†$ & 2373786 & 297 & 12.51 (11.17 to 14.02$)$ & 149817 & 21 & 14.02 (9.14 to 21.50 ) \\
\hline
\end{tabular}

Table shows outcomes with five or more vaccine exposed cases.

*Events per 100000 person years.

†Risk window for venous thromboembolism was within 90 days after vaccine exposure 
Table 3| Evaluation of signal strengthening criteria among outcomes where rate ratios were significantly increased

\begin{tabular}{|c|c|c|c|}
\hline Criterion & Behcet's syndrome & Raynaud's disease & Type 1 diabetes \\
\hline $\begin{array}{l}\text { Analysis based on } 20 \text { or more vaccine } \\
\text { exposed cases }\end{array}$ & No $(n=5)$ & Yes $(n=37)$ & Yes $(n=99)$ \\
\hline Rate ratio $\geq 3.0$ & Yes (3.37) & No (1.67) & No (1.29) \\
\hline $\begin{array}{l}\text { Significantly increased rate ratios in } \\
\text { both countries when analysed } \\
\text { separately }\end{array}$ & $\begin{array}{c}\text { No }\left(3.38^{\star}, 95 \% \text { Cl } 0.83 \text { to } 13.84 \text { for }\right. \\
\text { Sweden ( } 3 \text { exposed cases); } 4.63 \dagger, 95 \% \\
\text { Cl } 0.64 \text { to } 33.66 \text { for Denmark ( } 2 \text { exposed } \\
\text { cases)) }\end{array}$ & $\begin{array}{c}\text { No }\left(1.86^{*}, 95 \% \text { Cl } 1.19 \text { to } 2.89 \text { for }\right. \\
\text { Sweden ( } 25 \text { exposed cases); } 1.46^{*}, 95 \% \\
\text { Cl } 0.64 \text { to } 3.33 \text { for Denmark ( } 12 \text { exposed } \\
\text { cases)) }\end{array}$ & $\begin{array}{c}\text { No }\left(1.47^{\star}, 95 \% \mathrm{Cl} 1.08 \text { to } 2.01 \text { for }\right. \\
\text { Sweden ( } 47 \text { exposed cases); } 1.09^{*}, 95 \% \\
\text { Cl } 0.76 \text { to } 1.57 \text { for Denmark ( } 52 \text { exposed } \\
\text { cases)) }\end{array}$ \\
\hline
\end{tabular}

*Adjusted for age in two year intervals, calendar year, and parental country of birth, parental education, and paternal socioeconomic status. †Adjusted for age in two year intervals (model with full adjustment did not converge). 


\section{Figures}

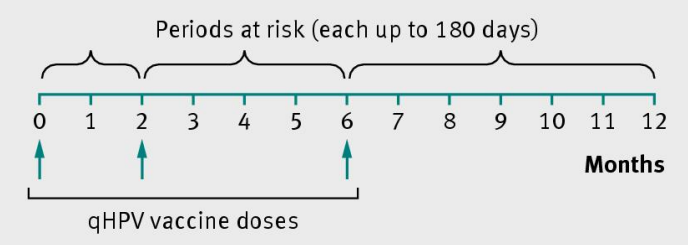

Fig 1 Periods at risk for autoimmune and neurological events in adolescent girls after exposure to quadrivalent human papillomavirus (qHPV) vaccine. For venous thromboembolism, each period at risk was up to 90 days

\begin{tabular}{|c|c|c|}
\hline $\begin{array}{l}\text { Event } \\
\text { Autoimmune }\end{array}$ & $\begin{array}{l}\text { Adjusted rate } \\
\text { ratio }(95 \% \mathrm{Cl})\end{array}$ & $\begin{array}{l}\text { Adjusted rate } \\
\text { ratio }(95 \% \mathrm{Cl})\end{array}$ \\
\hline \multicolumn{3}{|l|}{ Autoimmune } \\
\hline $\begin{array}{l}\text { Thyroid } \\
\text { Grave's disease }\end{array}$ & & \\
\hline Hashimoto's thyroiditis & - & 1.12 (0.82 to 1.52$)$ \\
\hline Other hyperthyroidism & $\longrightarrow$ & 0.99 (0.63 to 1.55$)$ \\
\hline Hypothyroidism & - & $0.90(0.71$ to 1.14$)$ \\
\hline \multicolumn{3}{|l|}{ Gastrointestinal } \\
\hline Coeliac disease & & 1.11 (0.90 to 1.36$)$ \\
\hline Crohn's disease & $\rightarrow$ & $0.85(0.62$ to 1.17$)$ \\
\hline Ulcerative colitis & & 0.71 (0.49 to 1.03$)$ \\
\hline Pancreatitis & & 1.19 (0.60 to 2.35$)$ \\
\hline \multicolumn{3}{|l|}{ Musculoskeletal/systemic } \\
\hline Ankylosing spondylitis & & 0.94 (0.44 to 2.01$)$ \\
\hline Behcets syndrome & & 3.37 (1.05 to 10.80$)$ \\
\hline Henoch-Schönlein's purpura & 一 & 0.89 (0.52 to 1.52$)$ \\
\hline Juvenile arthritis & & 0.99 (0.78 to 1.26$)$ \\
\hline Myositis & & $1.07(0.50$ to 2.31$)$ \\
\hline Rheumatoid arthritis & - & $1.01(0.66$ to 1.54$)$ \\
\hline Systemic lupus erythematosus & $\Rightarrow$ & 1.35 (0.69 to 2.67$)$ \\
\hline Vasculitis, unspecified & & 1.55 (0.83 to 2.88$)$ \\
\hline \multicolumn{3}{|l|}{ Haematological } \\
\hline Idiopathic thrombocytopenic purpura & - & $1.18(0.65$ to 2.17$)$ \\
\hline \multicolumn{3}{|l|}{ Dermatological } \\
\hline Erythema nodosum & - & 1.05 (0.63 to 1.73$)$ \\
\hline Localised scleroderma & & 1.04 (0.44 to 2.48$)$ \\
\hline Psoriasis & - & 1.01 (0.80 to 1.28$)$ \\
\hline Vitiligo & $\longrightarrow$ & $1.13(0.73$ to 1.74$)$ \\
\hline \multicolumn{3}{|l|}{ Miscellaneous } \\
\hline Raynaud's disease & $\longrightarrow$ & 1.67 (1.14 to 2.44$)$ \\
\hline Type 1 diabetes & 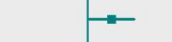 & 1.29 (1.03 to 1.62$)$ \\
\hline \multicolumn{3}{|l|}{ Neurological } \\
\hline Bell's palsy & 一 & $1.02(0.72$ to 1.43$)$ \\
\hline Epilepsy & $\rightarrow$ & 0.66 (0.54 to 0.80$)$ \\
\hline Narcolepsy & & 0.71 (0.29 to 1.79$)$ \\
\hline Optical neuritis & - & $0.67(0.27$ to 1.64$)$ \\
\hline Paralysis & - & $0.56(0.35$ to 0.90$)$ \\
\hline Venous thromboembolism & $\rightarrow$ & $0.86(0.55$ to 1.36$)$ \\
\hline & 1 & \\
\hline
\end{tabular}

Fig 2 Association between exposure to quadrivalent human papillomavirus (qHPV) vaccine and adverse events in adolescent girls in Denmark and Sweden, October 2006-December 2010. Rate ratios are adjusted for country, age in two year intervals, calendar year, and parental country of birth, parental education, and paternal socioeconomic status 

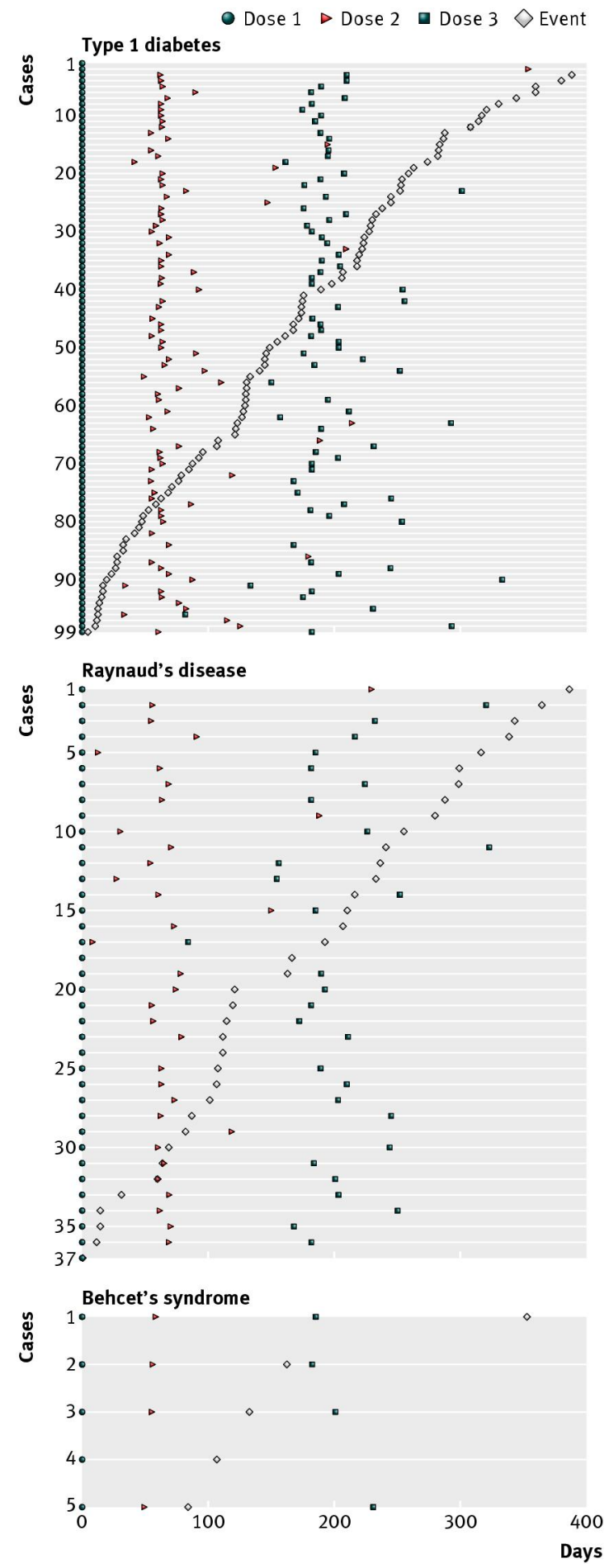

Fig 3 Distribution of cases according to days since first dose of quadrivalent human papillomavirus (qHPV) vaccine. For type 1 diabetes case 1, vaccine doses 2 and 3 and event are not displayed (dose 2 was administered on day 425 , dose 3 on day 609, and the event was on day 570). For type 1 diabetes case 2, event is not displayed (event was on day 460). For type 1 diabetes case 82 , vaccine dose 3 is not displayed (dose was administered on day 455) 